\title{
Rol de la cirugía mínimamente invasiva en la patología esofágica benigna: reporte de una serie de casos
}

\author{
ITALO BRAGHETTO M., OWEN KORN B., GONZALO CARDEMIL H., \\ HÉCTOR VALLADARES H., GONZALO MASIA L., CARLOS MANDIOLA B.
}

\section{Laparoscopic surgery for benign esophageal diseases}

Background: Minimally invasive surgery has the advantage of a lower rate of complications and can be used for benign esophageal diseases. Aim: To report a single surgeon experience with laparoscopic surgery for benign esophageal diseases. Material and Methods: Prospective analysis of 421 patients (160 males) with benign esophageal disease, who were subjected to laparoscopic surgery by a single surgeon. Immediate mortality, surgical complications and long term results in terms of symptoms recurrence, were analyzed. Results: The underlying diagnoses of the operated patients were Barrett's esophagus or esophagitis in 257, hiatal hernia in 91, achalasia in 68 and esophageal diverticula in five. Surgery obtained successful results in 90\% of patients with Barrett's esophagus. Among patients with hiatal hernia, there was a $12 \%$ rate of complications and a $30 \%$ recurrence, when a mesh was not used. Among patients with achalasia the recurrence rate was less than 5\%. Conclusions: Laparoscopic surgery has a fair success rate in benign esophageal diseases, with a lower rate of complications.

(Rev Med Chile 2012; 140: 703-712).

Key words: Barrett's esophagus; Esophageal diseases; Laparoscopy.

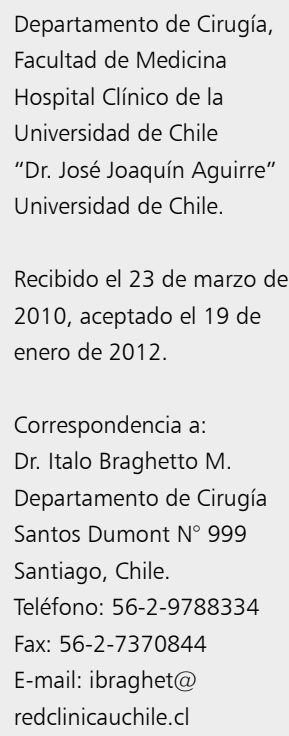

E n los últimos 15 años, la cirugía mínimamente invasiva ha mostrado grandes avances en la técnica quirúrgica y ha hecho posible la disminución de la morbimortalidad postoperatoria. Numerosos son los estudios que se han realizado en el seguimiento de patologías benignas susceptibles de ser abordadas por cirugía mínimamente invasiva, tales como enfermedad por reflujo gastroesofágico (RGE), hernia hiatal, divertículos esofágicos y acalasia, patologías que también en nuestro grupo de trabajo han sido tratadas con procedimientos mínimamente invasivos. Las indicaciones quirúrgicas para estas patologías están claramente establecidas en la literatura nacional e internacional y hemos seguido estrictamente estos protocolos ${ }^{1-3}$.

En este artículo, se analizan los resultados e indicaciones obtenidos por nuestro grupo en pacientes con patología esofágica benigna sometidos a diferentes técnicas quirúrgicas ya sea por vía laparoscópica o toracoscópica, y se comparan estos resultados con los resultados obtenidos en la era de la cirugía abierta publicados en la literatura internacional.

\section{Material y Método}

En este trabajo prospectivo se incluyeron un total de 421 pacientes consecutivos (160 hombres, 261 mujeres) con un promedio de edad de 49,8 años (rango entre 37 y 78 años) distribuidos de acuerdo a su patología esofágica y la técnica quirúrgica a la que fueron sometidos y que fueron operados exclusivamente por el autor (IB) del trabajo en los últimos 8 años. Todos estos pacientes se estudiaron de acuerdo a nuestro protocolo de 
estudio de la patología esofágica, lo que incluye endoscopia, biopsia, manometría, monitoreo de $\mathrm{pH}$ intraesofágico de 24 hrs y radiología baritada de acuerdo a los métodos previamente publicados ${ }^{4-6}$. Los procedimientos quirúrgicos empleados por el equipo quirúrgico se muestran en la Tabla 1 , según las técnicas descritas ampliamente en publicaciones anteriores para cada una de las patologías incluidas en este estudio ${ }^{7-9}$. Sólo se excluyeron de esta serie laparoscópica aquellos pacientes que habían sido sometidos previamente a intervenciones quirúrgicas laparoscópicas o abiertas por la misma patología. Las técnicas quirúrgicas empleadas para el reflujo gastroesofágico han sido previamente descritas, empleándose la fundoplicatura calibrada en aquellos pacientes con esofagitis o con esófago de Barrett, Barrett corto ${ }^{7}$ en pacientes con esófago de Barrett extenso o complicados con úlceras o estenosis se efectuó la fundoplicatura asociada a una técnica de supresión ácida y derivación biliar consistente en vagotomía troncal o selectiva más antrectomía y gastroyeyunostomía en Y-de-Roux con el objeto de evitar reflujo ácido y biliar presente en estos enfermos ${ }^{10,11}$.

Las hernias hiatales se clasifican de acuerdo al consenso internacional al respecto ${ }^{12}$, mientras que en tipo I, II, III y IV, con frecuencias de $90 \%$, $4 \%, 5 \%$ y $1 \%$ respectivamente. La hernia hiatal de tipo I o por deslizamiento axial, es aquella en la cual existe un desplazamiento de la UGE hacia el interior del mediastino a través del hiato esofágico, con saco herniario. La hernia hiatal de tipo II o paraesofágica, es aquella en la cual existe una herniación del fondo gástrico hacia el inte- rior del mediastino dentro de un saco herniario, manteniendo la posición correcta de la UGE intraabdominal. La hernia hiatal de tipo III, es una hernia mixta en la cual la UGE y una gran porción del estómago ha migrado hacia el interior del mediastino. Por último, la hernia hiatal de tipo IV, es aquella en la cual existe una gran hernia dentro del mediastino que contiene estómago, omento y a veces otras vísceras abdominales.

Se empleó el test de $\chi^{2}$ o test de de Fisher según los datos que se quisieran comparar. Se analizaron la morbimortalidad y los resultados alejados evaluados objetivamente: recurrencia de síntomas (pirosis o regurgitación, disfagia, dolor retro-esternal), hallazgos endoscópicos, manométricos o monitoreo de $\mathrm{pH}$ de $24 \mathrm{~h}$ anormal y radiológicos, de acuerdo al mismo protocolo preoperatorio y a la patología por la que fueron operados. La información de todos estos pacientes fue registrada en un base de datos (Excell) en la cual se registran todos los datos relacionados a diagnóstico, estudios endoscópicos, radiológicos, manométricos pre y postoperatorias, como también las complicaciones precoces y el seguimiento a largo plazo.

\section{Resultados}

En la Tabla 2 se presentan las complicaciones precoces postoperatorias clasificadas en mayores $y$ menores, en los pacientes con diferentes diagnósticos y tratamientos quirúrgicos empleados, en lo que se destaca la mínima tasa de complicaciones mayores y prácticamente nula mortalidad.

Tabla 1. Experiencia en patología esofágica no tumoral tratada con cirugía laparoscópica

\begin{tabular}{|lrl|}
\hline Patología & n pacientes & Técnica quirúrgica laparoscópica \\
Esofagitis / Barrett corto & 185 & Fundoplicatura calibrada + Gastropexia posterior + Cierre de pilares \\
E. de Barrett largo & 72 & $\begin{array}{l}\text { Fundoplicatura calibrada sola }(n=22) \\
\text { Fundoplicatura calibrada + Supresión ácida + Derivación biliar }(\mathrm{n}=29) \\
\text { GST 95\%+ gastroyeyunostomía Y de Roux asa larga }(\mathrm{n}=21)\end{array}$ \\
$\begin{array}{l}\text { Hernia hiatal } \\
\text { Acalasia }\end{array}$ & 61 & $\begin{array}{l}\text { Fundoplicatura calibrada + hiatoplastía } \\
\text { Sin malla }(\mathrm{n}=68) \text { Con malla }(\mathrm{n}=23)\end{array}$ \\
$\begin{array}{l}\text { Divertículo mediotorácico } \\
\text { Divertículo epifrénico }\end{array}$ & 1 & Cardiomiotomía + Fundoplicatura de Dor \\
Total & 4 & Diverticulectomía + Miotomía + Fundoplicatura de Dor \\
\hline
\end{tabular}


Tabla 2. Complicaciones precoces post cirugía laparoscópica por patología esofágica benigna

\begin{tabular}{|c|c|c|c|c|c|}
\hline \multirow[b]{2}{*}{ Diagnóstico } & \multirow[b]{2}{*}{ Técnica quirúrgica } & \multicolumn{4}{|c|}{ Complicaciones precoces } \\
\hline & & $\mathbf{n}$ & Mayores & Menores & Mortalidad \\
\hline ERGEP & Fundoplicatura + Gastropexia + Cierre pilares & 185 & 7 disfagia & 0 & 0 \\
\hline \multirow[t]{3}{*}{ Barrett } & Fundoplicatura sola & 22 & 1 * & 0 & 0 \\
\hline & + Supresión ácida/Derivación biliar & 29 & $2^{* *}$ & 0 & 0 \\
\hline & Bypass gástrico resectivo & 21 & $1 * * *$ & 0 & 0 \\
\hline \multirow[t]{2}{*}{ Hernia hiatal } & Hernioplastia s/malla + fundoplicatura c/malla & 91 & 6 & 5 & 0 \\
\hline & Acalasia + Parche de Dor & 68 & 3 & $1 * * * *$ & 0 \\
\hline \multirow[t]{2}{*}{ Divertículos } & Diverticulectomía Esofágicos + & 4 & & $1 * * * * *$ & 0 \\
\hline & $\mathrm{N}^{\circ}$ total pacientes & 421 & $20(47 \%)$ & & 0 \\
\hline
\end{tabular}

${ }^{*}$ Disfagia severa. ${ }^{* *}$ Disfagia severa-Fístula muñón duodenal. ${ }^{* * *}$ Hemorragia digestión auto limitada. ${ }^{* * *}$ Neumoperitoneo. $* * * *$ Colección subfrénica.

\section{Enfermedad por reflujo gastroesofágico (RGE) y esófago de Barrett \\ Cirugía antirreflujo se efectuó en 185 pacien-} tes con esofagitis grado A-B de la clasificación de Los Ángeles y sin esófago de Barrett. Éxito sintomático se observó sobre el $90 \%$ y una esofagitis persistente o recurrente en $15 \%$, manometría anormal en $14 \%$ de los casos y el monitoreo de $\mathrm{pH}$ intraesofágico de $24 \mathrm{~h}$ mostró persistencia de reflujo ácido en 19,5\%, lo que demuestra que muchos pacientes aun siendo asintomáticos presentan un esfínter hipotenso y reflujo ácido postoperatorio, siendo estos pacientes en su gran mayoría portadores de esófago de Barrett. Los pacientes con esófago de Barrett $(n=72)$ merecen un análisis aparte. Hay 3 grupos de pacientes, en 22 de ellos con esófago de Barrett corto $(<$ de 3 $\mathrm{cms}$ de longitud) se efectúa calibración cardial y fundoplicatura, y en otros 28 con esófago de Barrett extenso o complicado (presencia de úlcera o estenosis), además de la fundoplicatura, se efectúa un procedimiento de supresión ácida y derivación biliar mediante vagotomía, antrectomía más gastroyeyunostomía en Y de Roux. Se incluye un tercer grupo de pacientes que teniendo esófago de Barrett presentan además obesidad mórbida. En estos pacientes se efectuó una gastrectomía casi total (95\%) con gastroyeyunostomía en Y de Roux con la finalidad de tratar ambas patologías. Los pacientes con esófago de Barrett extenso o complicado operados con fundoplicatura clásica, presenta malos resultados con alta recurrencia de los síntomas de reflujo, esofagitis endoscópica, in- competencia del esfínter gastroesofágico y reflujo ácido postoperatorio anormal en comparación a los resultados postoperatorios de los pacientes sometidos a supresión ácida y derivación biliar adicionada a la fundoplicatura. Todos estos pacientes tienen más de 3 años de seguimiento (rango: 3-8 años) (Tabla 3).

\section{Hernia hiatal $(\mathrm{HH})$}

La indicación de cirugía en estos pacientes se debió a que todos ellos presentaban síntomas secundarios a reflujo gastroesofágico severo, síntomas respiratorios algunos de ellos con cuadros de broncoaspiración, neumonitis a repetición, síntomas cardiovasculares asociados al gran tamaño de su hernia, en general mayores de 5 $\mathrm{cm}$ en ocasiones con vólvulo gástrico con mal vaciamiento del estómago.

Se operaron 91 pacientes, 43 casos de hernia hiatal tipo I, 5 casos de tipo II, 28 casos de tipo III y 15 casos de tipo IV. Complicaciones postoperatorias ocurrieron en 11 pacientes $(13,1 \%)$, siendo las más frecuentes derrame pleural en 3 pacientes, disfagia postoperatoria que necesitó dilatación en 4 pacientes, atonía gástrica transitoria en 2 pacientes, 1 paciente con hemoperitoneo y 1 paciente con hemorragia digestiva alta por úlcera gástrica. No hubo mortalidad postoperatoria en esta serie.

Las recidivas fueron evaluadas en función de un estudio radiológico pre y post operatorio. En total hubo 12 casos de recidiva entre los cuales se encontraba 3 casos de los 43 de hernia hiatal tipo 
Tabla 3. Falla sintomática y objetiva en pacientes con esofagitis sin Esófago de Barrett sometidos a fundoplicatura y pacientes con esofagitis y Barrett sometidos a diferentes procedimientos quirúrgicos por vía laparoscópica

\begin{tabular}{|c|c|c|c|c|c|}
\hline & $\begin{array}{l}\text { Síntomas } \\
\text { recurrentes } \\
\text { n (\%) }\end{array}$ & $\begin{array}{c}\text { Reflujo } \\
\text { radiológico } \\
\text { n (\%) }\end{array}$ & $\begin{array}{c}\text { Esofagitis } \\
\text { endoscópica } \\
n(\%)\end{array}$ & $\begin{array}{c}\text { Esfínter } \\
\text { incompetente } \\
n(\%)\end{array}$ & $\begin{array}{c}\text { Refllujo ácido* } \\
\text { anormal } \\
\text { n (\%) }\end{array}$ \\
\hline $\begin{array}{l}\text { A) Pacientes con esofagitis o Esófago de } \\
\text { Barrett corto }(n=185)\end{array}$ & $18(9,7)$ & $14(7,6)$ & $27(14,6)$ & $21(11,3)$ & $\begin{array}{l}36(19,5) \\
(P<0,05)\end{array}$ \\
\hline \multicolumn{6}{|l|}{ B) Pacientes con esófago de Barrett } \\
\hline a. Fundoplicatura calibrada $(n=22)$ & $8(36,4)$ & $6(27,3)$ & $11(50)^{* *}$ & $7(31,8)$ & $\begin{array}{c}9(40,9) \\
(P<0,05)\end{array}$ \\
\hline $\begin{array}{l}\text { b. Fundoplicatura calibrada }+ \text { supresión } \\
\text { ácida }+ \text { derivación biliar }(n=29)\end{array}$ & $1(3,6)$ & $1(3,6)$ & $1(3,6)$ & $6(21,4)$ & $1(3,6)$ \\
\hline $\begin{array}{l}\text { c. GST } 95 \%+\text { Gastroyeyunostomía en } Y \\
\text { de Roux }(n=21)\end{array}$ & 0 & 0 & 0 & 21 & 2 \\
\hline
\end{tabular}

*Monitoreo de pH intraesofágico de 24 hr. **11 esofagitis erosiva, 4 úlcera esofágica, 1 progresión a displasia.

I, 5 casos en pacientes con hernia hiatal tipo III y 4 casos de los 15 con hernia hiatal tipo IV. Los pacientes en los cuales se ha usado malla de refuerzo la recurrencia de la hernia es significativamente menor (Tablas 4 y 5 ).

\section{Acalasia esofágica}

Del total de pacientes, en tres casos existieron complicaciones intraoperatorias por daño de la mucosa esofágica, siendo suturadas de inmediato en dos de ellos, con necesidad de conversión sólo en un caso. Se observó un caso de neumoperitoneo que se trató sólo con drenaje percutáneo y la estadía hospitalaria fue de 3 días (Tabla 2). En 15 de 68 (22\%) pacientes se observó síntomas de RGE postoperatorio y en todos ellos se encontró reflujo ácido positivo, presentando uno de ellos signos de esofagitis erosiva al estudio endoscópico. Del total de pacientes operados, sólo en cinco de ellos $(7,3 \%)$ se ha precisado de un tratamiento de dilatación endoscópica única dada la presencia de disfagia post quirúrgica, con resultados satisfactorios. Los resultados clínicos y funcionales de 68 pacientes controlados se muestran en la Tabla 6, confirmándose excelentes resultados en cuanto a normalización de la presión de reposo y de la relajación del esfínter esofágico inferior
Tabla 4. Experiencia en 91 pacientes con hernia hiatales operados por vía laparocópica. Tipo de hernia y recidiva postoperatoria

\begin{tabular}{|ccc|}
\hline $\begin{array}{c}\text { Tipo de } \\
\text { hernia hiatal }\end{array}$ & $\begin{array}{c}\text { n de } \\
\text { pacientes }\end{array}$ & $\begin{array}{c}\text { n de } \\
\text { recidivas }\end{array}$ \\
Tipo I & $43 \longrightarrow 3$ \\
\hline Tipo II & 5 & 4 \\
Tipo III & 28 & 5 \\
\hline Tipo IV & 15 & - \\
\hline Total & 91 & $12(12,3 \%)$ \\
\hline
\end{tabular}

Tabla 5. Recurrencia según tipo de hernia con y sin malla de refuerzo

\begin{tabular}{|c|c|c|c|c|c|c|c|}
\hline \multirow[t]{2}{*}{ Malla } & \multicolumn{4}{|c|}{ Tipo } & \multirow[t]{2}{*}{ Total } & \multirow{2}{*}{$\begin{array}{l}\text { Recu- } \\
\text { rrencia }\end{array}$} & \multirow[t]{2}{*}{$\mathbf{p}$} \\
\hline & $\begin{array}{c}\text { I } \\
(\operatorname{Rec})\end{array}$ & $\begin{array}{c}\text { II } \\
(\operatorname{Rec})\end{array}$ & $\begin{array}{c}\text { III } \\
(\operatorname{Rec})\end{array}$ & $\begin{array}{c}\text { IV } \\
(\operatorname{Rec})\end{array}$ & & & \\
\hline Sí & - & (0) & $\begin{array}{l}14 \\
(0)\end{array}$ & $\begin{array}{l}8 \\
(0)\end{array}$ & 23 & 0 & \\
\hline No & $\begin{array}{l}43 \\
(3)\end{array}$ & $\begin{array}{c}4 \\
(0)\end{array}$ & $\begin{array}{l}14 \\
(5)\end{array}$ & $7(4)$ & 68 & $\begin{array}{c}12 \\
(17,9 \%)^{*}\end{array}$ & $(0,03)$ \\
\hline Total & $\begin{array}{l}43 \\
(3)\end{array}$ & $\begin{array}{c}5 \\
(0)\end{array}$ & $\begin{array}{l}28 \\
(5)\end{array}$ & $\begin{array}{l}15 \\
(4)\end{array}$ & 91 & $\begin{array}{c}12 \\
(12,3 \%)\end{array}$ & \\
\hline
\end{tabular}


y disminución del diámetro del cuerpo esofágico al obtenerse mejoría del vaciamiento esofágico.

\section{Divertículos esofágicos}

En nuestro grupo se trató 1 paciente portador de una fístula esófago-bronquial secundaria a un divertículo medio torácico abordado por vía transtorácica, resecando el divertículo y realizando sutura tanto del lado bronquial como del cuello esofágico del divertículo y 4 pacientes con divertículos epifrénicos, trata- dos con resección del divertículo y miotomía del esfínter esofágico inferior abordados por vía transhiatal (Figuras 1 y 2). Sólo hubo una complicación postoperatoria derivada de una pequeña fuga localizada de la línea de sutura que fue tratada medicamente (Tabla 2).

Tabla 6. Resultados manométricos en el tratamiento quirúrgico de la acalasia esofágica

\begin{tabular}{|lccc|}
\hline Manometría & Preop $\overline{\mathbf{x}} \pm$ DS & Postop $\overline{\mathbf{x}} \pm$ DS & P* \\
- Presión EGE (mmHg) & $33,5 \pm 9,09$ & $9,8 \pm 3,5$ & 0,000 \\
- Amplitud ondas & $29,2 \pm 11,3$ & $35,8 \pm 14,2$ & $\mathrm{~ns}$ \\
- \% Relajación EGE & $56,2 \pm 22,3$ & $100 \pm 0,0$ & 0,000 \\
Radiología (diámetro mm) & & & \\
- EGE & $2,0 \pm 2,5$ & $15,1 \pm 3,9$ & 0,000 \\
- Cuerpo & $51,4 \pm 16,3$ & $30,0 \pm 3,4$ & 0,000 \\
\hline
\end{tabular}
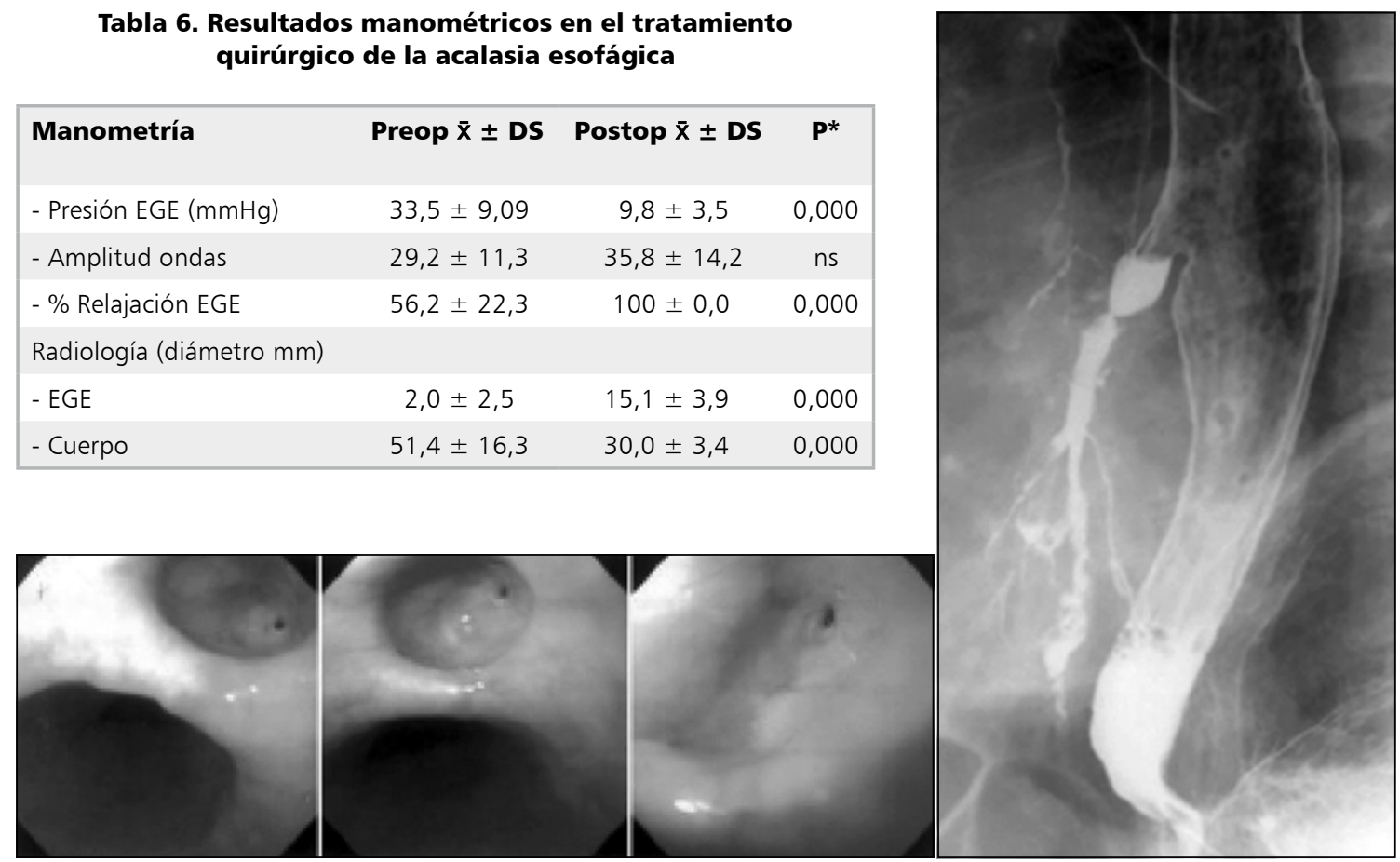

Figura 1. Visión endoscópica y radiológica del divertículo mediotorácico y fístula broncoesofágica.
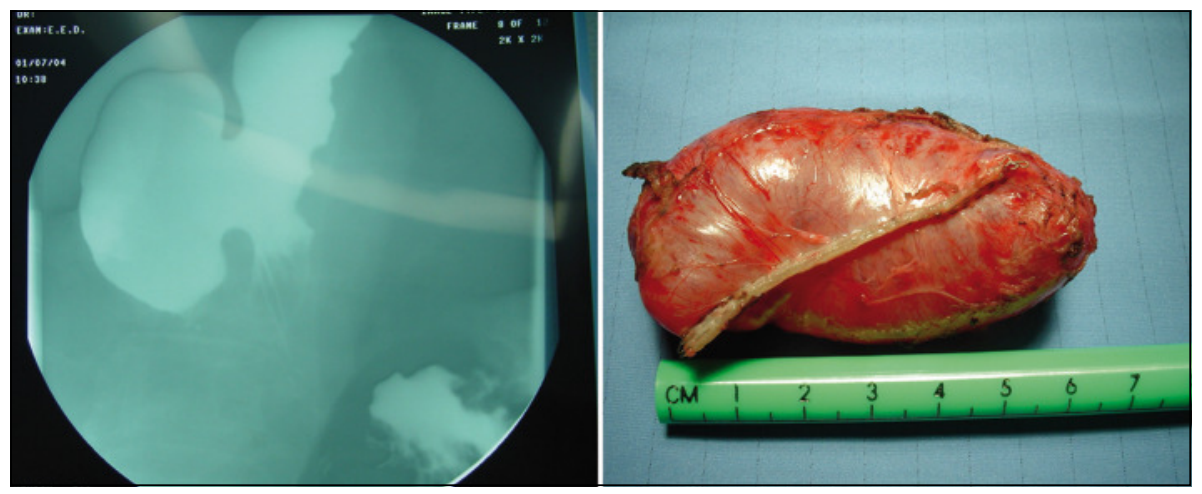

Figura 2. Divertículo epifrénico y pieza resecada por vía laparoscópica transhiatal. 


\section{Discusión}

\section{Enfermedad por reflujo gastroesofágico y esófago de Barrett}

Los procedimientos mínimamente invasivos en la cirugía antirreflujo son actualmente aceptados como gold standard para el tratamiento quirúrgico de pacientes con esofagitis por RGE, cuya indicación quirúrgica cumpla con los requisitos conocidos internacionalmente (Consenso Montreal) ${ }^{13}$. La fundoplicatura de Nissen, la fundoplicatura modificada de Nissen-Rosetti o la fundoplicatura parcial con las técnicas de Toupet o de Lind, han sido los procedimientos quirúrgicos más comúnmente utilizados ${ }^{6-9}$. En nuestra experiencia, la fundoplicatura calibrada más gastropexia posterior ha sido evaluada a corto y largo plazo, mostrando resultados equivalentes a los otros procedimientos con resultados alejados ampliamente aceptados en la literatura mundial ${ }^{14-16}$.

En pacientes con esófago de Barrett corto no complicado más de $75 \%$ de los pacientes presentan excelentes resultados sintomáticos. Sin embrago, en pacientes con EB extenso o complicado, con úlcera o estenosis sometidos a fundoplicatura de Nissen, la recurrencia es muy frecuente, siendo necesario medicación antirreflujo en un alto porcentaje de ellos, la recurrencia de reflujo ácido persistente es de $25 \%$ de los pacientes.

En una revisión de la literatura, se ha reportado que $2,5 \%$ de pacientes operados por RGE sin esofagitis (grupo control) deben ser sometidos a cirugía de revisión, en cambio $6,5 \%$ de los pacientes con esofagitis sin Barrett y $12 \%$ de los pacientes con EB debieron ser reoperados ${ }^{17-19}$.

De acuerdo a nuestra conducta ${ }^{18}$ de controlar tanto el reflujo ácido como biliar, factores importantes en la patogenia de la enfermedad, hemos operado 29 pacientes con EB extenso complicado, realizando una técnica antirreflujo gastroesofágico asociado con un procedimiento de supresión ácida y derivación duodenal (vagotomía + antrectomía + gastroyeyunostomía en Y de Roux) por vía laparoscópica, demostrando ser una técnica factible, sin mayores complicaciones, lo que actualmente está siendo aceptado en la litera- tura quirúrgica internacional ${ }^{20}$. En 21 pacientes con EB y obesidad asociada hemos efectuado una resección gástrica 95\% con anastomosis gastroyeyunal en $Y$ de Roux con un asa de 150-170 cm de largo (asa alimentaria) con excelentes resultados sintomáticos y desaparición de la esofagitis. Csendes y otras publicaciones recomiendan esta técnica para estos pacientes resolviendo el problema de su obesidad y de los síntomas derivados del $\mathrm{RGE}^{20-27}$. Sin embargo, este punto no está aún dilucido y existe gran controversia entre gastroenterólogos y cirujanos dedicados al tema. De acuerdo a recientes publicaciones tanto el tratamiento médico como la fundoplicatura pueden ser opciones recomendables para el control de la exposición al ácido del esófago distal, pero no mencionan técnicas de supresión ácida y derivación biliar para control de ambos componentes de reflujo ${ }^{28}$.

\section{Hernias hiatales}

La indicación quirúrgica de las hernias hiatales está dada por su gran asociación con enfermedad por RGE (cerca de 70\%), y complicaciones, tales como vólvulo o incarceración del contenido con obstrucción, lo que puede traducirse como disfagia, vómitos, dolor retroesternal, disnea o broncoaspiración. Cuando esto se asocia a hemorragia digestiva alta, se debe sospechar en isquemia, estragulación o perforación del órgano, que son complicaciones asociadas a una alta morbilidad y mortalidad ${ }^{29-40}$. La evidencia actual indica realizar el procedimiento antirreflujo, debido a que una gran mayoría de pacientes tienen enfermedad por RGE. Existe tendencia hacia el uso de malla en hernias de más de $5 \mathrm{~cm}$ de diámetro en base a que existe una mayor recurrencia de $\mathrm{HH}$ en los grupos de pacientes en que no se ha utilizado (19\%) versus los grupos en que sí se ha utilizado (0-5\%) (Tabla 7).

Tabla 7. Cirugía laparoscópica en hernia hiatal: revisión de 20 trabajos seleccionados

\begin{tabular}{|c|c|c|}
\hline Año & & $1997-2005$ \\
\hline Pacientes & & 1.415 (33-203 pacientes incluidos) \\
\hline Conversión & & $3,1 \%\left(0^{*}-9,1 \%\right)$ \\
\hline Edad & & 65,7 años (18 - 102) \\
\hline Estadía hospitalaria & & 2,7 días $(2-4)$ \\
\hline Cirugía anti RGE & & $94 \%$ \\
\hline Uso de malla & $\begin{array}{l}\text { No } \\
\text { Sí }\end{array}$ & $\begin{array}{l}9 \text { autores } \\
11 \text { autores }\end{array}$ \\
\hline Resección saco & & $90 \%$ \\
\hline
\end{tabular}

*3 Trabajos. 
De la revisión de la literatura publicada entre los años 1995 y 2003 en un total de 764 pacientes operados, las complicaciones se desarrollaron en $18,1 \%$ (12-28\%), síntomas postoperatorios persistieron en $13,3 \%(8-21 \%)$ y la recurrencia se desarrolló en el $11,9 \%(0-42 \%)$ de los casos. De los tres estudios que utilizaron malla (121 pacientes), sólo uno presentó $5 \%$ de recurrencias (Tabla 8$)^{35-40}$.

\section{Acalasia esofágica}

Diversos estudios han comparado la eficacia de las terapias médicas respecto a los resultados ofrecidos por las distintas técnicas quirúrgicas en el enfrentamiento de esta enfermedad, con pobres resultados a largo plazo ${ }^{41-44}$. Es así como la cardiomiotomía anterior se ha convertido en el tratamiento de elección de la acalasia esofágica, al ser más efectiva en mejorar la disfagia, presentando mínimas tasas de complicaciones intraoperatorias y conversión, un menor tiempo de estadía intrahospitalaria y excelentes resultados a

Tabla 8. Complicaciones post operatorias en cirugía laparoscópica de las hernias hiatales: revisión de la literatura $(n=1.366$ pacientes)

\begin{tabular}{|lc|}
\hline Intraoperatorias & $5,3 \%(0-10 \%)$ \\
\hline Postoperatorias** & $12,7 \%(0-45 \%)$ \\
\hline $\begin{array}{l}\text { Reoperación } \\
\text { - Precoz }\end{array}$ & $1,2 \%$ \\
- Tardías (> 30 días) & $3,0 \%$ \\
\hline Mortalidad & $1,1 \%(15 / 1.366$ pacientes) \\
- Perforación o necrosis & 10 pacientes \\
- Complic. cardiorrespiratorias & 5 pacientes \\
\hline
\end{tabular}

**Gran porcentaje de complicaciones son menores. largo plazo con tasa de recurrencia de la enfermedad menos de $5 \%{ }^{45-48}$.

La recurrencia de la enfermedad, hernias hiatales paraesofágicas o reflujo gastroesofágico asociado o no a esofagitis en el postoperatorio alejado varían entre 10 y $25 \%$ de frecuencia ${ }^{39-44}$. Se han reportado trabajos que consideran modificaciones a la técnica antes mencionada con el objeto de evitar o reducir las complicaciones ya descritas ${ }^{48}$.

\section{Patología diverticular del esófago}

La patología diverticular del esófago considera la existencia de divertículos de Zenker; divertículos medio torácicos y divertículos epifrénicos.

En los pacientes con divertículo de Zenker la diverticulectomía con miotomía cricofaríngea cuyos resultados ha exhibido tasas de resolución de los síntomas superior a 95\%. Las complicaciones varían entre 10 y 30\%, asociándose a una mortalidad mínima ${ }^{45-53}$. Los procedimientos endoscópicos presentan una tasa promedio de complicaciones de 5,5\%, y una tasa de mortalidad promedio de $0,2 \%$, con recurrencia de la sintomatología de 6,6\% para el grupo de técnicas vía endoscópica ${ }^{49-57}$.

En cuanto a la enfermedad diverticular que compromete el tercio medio y distal del esófago, en general, para los divertículos medio torácicos la indicación quirúrgica corresponde a una diverticulectomía realizada por vía transtorácica. En el caso de los divertículos epifrénicos sintomáticos grandes (Figura 2) tienen indicación de una diverticulectomía transhiatal junto a una miotomía distal ${ }^{58,59}$.

Tabla 9. Comparación resultados cirugía laparoscópica vs cirugía abierta en el Hospital Clínico de la Universidad de Chile*

\begin{tabular}{|lcccccc|}
\hline & \multicolumn{2}{c}{$\begin{array}{c}\text { Complicaciones } \\
\text { postoperatorias } \\
\text { Lap }\end{array}$} & \multicolumn{2}{c}{ Mortalidad } & \multicolumn{2}{c|}{ Seguimiento \% éxito } \\
\hline Abierta & Lap & Abierta & Lap & Abierta \\
\hline $\begin{array}{l}\text { Fundoplicatura sola } \\
\text { Derivación duodenal }\end{array}$ & $0,4 \%$ & 0 & $0 \%$ & $95 \%$ & $85 \%$ & \\
Hernioplastia hiatal & $10,3 \%$ & $7 \%$ & 0 & $1,5 \%$ & $97 \%$ & $91 \%$ \\
Cardiomiotomía + Dor & $13,6 \%$ & $15 \%$ & 0 & $1,4 \%$ & $82 \%$ & $88 \%$ \\
Diverticulectomía & $0,6 \%$ & $1,2 \%$ & 0 & 0 & $94 \%$ & $92 \%$ \\
\hline
\end{tabular}




\section{Conclusión}

En definitiva, las técnicas de cirugía mínimamente invasiva diseñadas para el abordaje de los diversos tipos de patologías que afectan al esófago, resultan por lo general altamente factibles observándose disminución de las complicaciones en comparación a la cirugía abierta, con iguales resultados a largo plazo (Tabla 9).

Es indudable, que para el adecuado uso de las distintas técnicas de cirugía mínimamente invasiva y la maximización de sus ventajas, es imprescindible un correcto estudio y selección de los pacientes, evaluando continuamente los resultados, siendo para ello mandatorio el mejoramiento continuo del entrenamiento y adquisición de destrezas, junto al desarrollo del mejor criterio clínico.

\section{Referencias}

1. Watson DL, Jamieson GG. Antireflux surgery in the laparoscopic era. Br J Surg 1998; 85: 1173-84.

2. Peters JH, Heimbucker J, Kauer WK, Incarbone R, Bremmene CG, DeMeeser TR. Clinical and physiologic comparison of laparoscopic and open Nissen fundoplication. J Am Coll Surg 1995; 180: 385-93.

3. Hinder RA, Filipi CJ, Wetscher G, Neary P, DeMeester TR, Perdikis G. Laparoscopic Nissen fundoplication is an effective treatment for gastroesophageal reflux disease. Ann Surg 1994; 220: 472-83.

4. Csendes A, Braghetto I, Korn O, Burdiles P. Late subjective and objective evaluation of antireflux surgery in patients with reflux esophagitis: analysis of 215 patients. Surgery 1999; 105: 374-82.

5. Csendes A, Larraín A. Effect of posterior gastropexy on gastroesophageal sphincter pressure and symptomatic reflux in patients with hiatal hernia. Gastroenterolgy 1972; 63: 19-20.

6. Csendes A, Miranda M, Espinoza M. Perimeter and location of the muscular gastroesophageal junction or "cardia" in control subjects and in patients with reflux esophagitis or achalasia. Scand J Gastroenterol 1981; 16: 951-6.

7. Braghetto I, Korn O, Debandi A, Burdiles P, Valladares $\mathrm{H}$, Csendes A. Laparoscopic cardial calibration and gastropexy for treatment of patients with reflux esophagitis: pathophysiological basis and result. World J Surg 2005; 29: 636-44.

8. Braghetto I, Korn O, Burdiles P, Debandi A, Valladares H. Hernias hiatales verdaderas: tratamiento quirúrgico por vía laparoscópica. Rev Chil Cir 2002; 54: 628-38.

9. Braghetto I, Korn O, Valladares H, Rodríguez A, Debando A, Brunet L. Laparoscopic anterior cardiomyotomy plus Anterior Dor fundoplication without division of lateral and posterior periesophageal anatomic structures for treatment of achalasia of the esophagus. Surg Laparosc Endosc Percutan Tech 2007; 17: 369-74.

10. Braghetto I, Korn O, Valladares H, Debandi A, Díaz JC, Brunet L. Laparoscopic surgical treatment for patients with short and long segment Barrett's esophagus. Which technique in which patient? Intern Surgery 2011; 96: 95-103.

11. Braghetto I, Csendes A, Smok G, Gradiz M, Mariani V, Compan A, et al. Histological inflammatory changes after surgery at the epithyelium of the distal esophagus in patients with Basreett's esophagus. Dis Esophagus 2004; 17: 235-42.

12. Braghetto I, Korn O, Csendes A, Burdiles P, Valladares $\mathrm{H}$, Brunet L. Postoperative results after laparoscopic approach for treatment of large hiatal hernias: Is mesh always need? Is the addition of an antireflux procedure necessary. Int. Surg 2010; 95: 80-7.

13. Stefanidis D, Hope WW, Kohn GP, Reardom PR, Richardson WS, Fanelli RD. Sages Guideline Committee. Guidelines for surgical treatment of gastroesophageal reflux disease. SAGES, 2010. (www.sages.org/org).

14. Geagea T. Laparoscopic Nissen-Rossetti fundoplication. Surg Endosc 1994; 8: 1080-4.

15. Bell RC, Hanna P, Powers B, Sabel Jr, Hruza D. Clinical and manometric results of laparoscopic parcial (Toupet) and complete (Nissen-Rossetti) fundoplication. Surg Endosc 1996; 10: 724-8.

16. Crookes PF, DeMeester TR. Complete and partial laparoscopic fundoplication for gastroesophageal reflux disease. Surg Endosc 1997; 11: 613-4.

17. Csendes A. Surgical treatment of Barrett's esophagus: 1980-2003. World J Surg 2004; 28: 225-31.

18. Braghetto I, Csendes A, Puente J, Burdiles P, Korn O. Esófago de Barrett. Rev Chil Cir 1997, 49: 119-29.

19. Spechler SJ, Lee E, Ahnen D, Goyal RK, Hirano I, Ramirez F, et al. Long-term outcome of medical and surgical therapies for gastroesophageal reflux disease: follow-up of a randomized controlled trial. JAMA 2001; 285: 23318.

20. Peters JH, Wang KK. How should Barrett's ulceration be treated? Surg Endosc 2004; 18: 338-44.

21. Jamieson GC, France M, Watson DI. Results of laparoscopic antireflux operations in patients who have Barrett's esophagus. Chest Surg Clin N Am 2002; 12: 149-55.

22. Farrell TM, Smith CD, Metreveli RE, Johnson AB, 
Galloway KD, Hunter JG. Fundoplication provides effective and durable symptom relief in patients with Barrett's esophagus. Am J Surg 1999; 78: 18-21.

23. Cobey F, Oeschlager B. Complete egression of Barrett's esophagus after Roux-en-Y gastric bypass. Obesity Surg 2005; 15: 710-2.

24. Kamolz T, Granderath F, Pointner R. Laparoscopic antireflux surgery: disease-related quality-of-life assessment before and after surgery in GERD patients with and without Barrett's esophagus. Surg Endosc 2003; 17: 880-5.

25. Yau P, Watson DI, Devitt PG, Game PA, Jamieson GG. Laparoscopic antireflux surgery in the treatment of gastroesophageal reflux in patients with Barrett's esophagus. Arch Surg 2000; 135: 801-5.

26. Braghetto I, Csendes A, Burdiles P, Korn O. Antireflux surgery, highly selective vagotomy and duodenal switch procedure: postoperative evaluation in patients with complicated and non complicated Barrett's esophagus. Dis Esoph 2000; 13: 12-7.

27. Mejía-Rivas MA, Herrera-López A, Hernández-Calleros J, Herrera M, Valdovinos MA. Gastroesophageal Reflux Disease in Morbid Obesity: The Effect of Roux-en-Y. Gastric Bypass. Obes Surg 2008; 18: 1211.

28. American Gastroenterological Association Medical Position Statement on the Management of Barrett's esophagus. Gastroenterology 2011; 140: 1084-91.

29. Wolf BS. Sliding hiatal hernia: the need for redefinition. Am J Roentgent 1973; 117: 231-9.

30. Rodefeld MD, Soper NJ. Parahiatal hernia with volvulus and incarceration: laparoscopic repair of a rare defect. J Gastrointest Surg 1998; 2: 193-7.

31. Hiebert CA. Massive hiatal hernia in esophageal surgery. In: Pearson C, et al. 2nd ed. New York: Livingstone-Churchil 2002; 279-84.

32. Landreneau RJ, Johnson JA, Marshall JB, Hazelrigg SR, Boley TM, Curtis JJ. Clinical spectrum of paraesophageal herniation. Dig Dis Sci 1992; 37: 537-44.

33. Wo JM, Branum GD, Hunter JG, Trus TN, Mauren SJ, Waring JP. Clinical features of type III (mixed) paraesophageal hernia. Am J Gastroent 1996; 91: 914-6.

34. Swanstrom LL, Jobe BA, Kinzie LR, Horvath KD. Esophageal motility and outcomes following laparoscopic paraesophageal hernia repair and fundoplication. Am J Surg 1999; 177: 359-63.

35. Hashemi M, Peters JH, DeMeester TR, Huprich JE, Quek M, Hagen JA, et al. Laparoscopic repair of large type III hiatal hernia: objective follow up reveals high recurrence rate. J Am Coll Surg 2000; 190: 553-61.

36. Díaz S, Brunt LM, Klingensmith ME, Friselle PM, Sope NJ. Laparoscopic paraesophageal hernia repair, a challenging operation: medium-term outcome of 116 patients. J Gastrointest Surg 2003; 7: 59-67.

37. Van der Peet DL, Kinkenberg-Knol EC, Alonso Poza A, Sietses C, Eijsbouts QA, Cuesta MA. Laparoscopic treatment of large paraesophageal hernias: both excision of the sac and gastropexy are imperative for adequate surgical treatment. Surg Endosc 2000; 14: 1015-8.

38. Oddsdottir M. Paraesophageal hernia. Surg Clin N A 2000; 80: 1243-52.

39. Mehta MA, Boddy AP, Rhodes M. Review of outcome after laparoscopic paraesophageal hiatal hernia repair. Surg. Lap. Endosc. Perc Tech 2006; 16: 301-6.

40. Braghetto I, Korn O, Csendes A, Burdiles P, Valladares $\mathrm{H}$, Brunet L. Postoperative Results After Laparoscopic Approach for Treatment of Large Hiatal Hernias: Is Mesh always needed? Is the Addition of an Antireflux Procedure Necessary? International Surg 2010; 95: 80-7.

41. Csendes A, Braghetto I, Henríquez A. Late results of a prospective randomized study comparing forceful dilatation and esophagomyotomy in patients with achalasia. Gut 1989; 30: 299-304.

42. Zaninotto G, Annese V, Costantini M, Del Genio A, Costantino M, Epifani M, et al. Randomized controlled trial of botulinum toxin versus laparoscopic Heller myotomy for esophageal achalasia. Ann Surg 2004; 239: 364-70.

43. Gockel I, Junginger T, Eckardt VF. Effects of pneumatic dilatation and myotomy on esophageal function and morphology in patients with achalasia. Am Surg 2005; 71: 128-31.

44. Braghetto I, Csendes A, Burdiles P, Korn O, Valera JM. [Current management of achalasia of the esophagus: critical review and clinical experience]. Rev Med Chile 2002; 130: 1055-66.

45. Pellegrini CA, Leichter R, Patti M, Somberg K, Ostroff JW, Way L. Thorascopic esophageal myotomy in the treatment of achalasia. Ann Thorac Surg 1993; 56: $680-2$.

46. Robinson TN, Galvani CA, Dutta SK, Gorodner MV, Patti MG. Laparoscopic treatment of recurrent dysphagia following transthoracic myotomy for achalasia. J Laparoendosc Surg Adv Tech 2003; 13: 401-3.

47. Csendes A, Braghetto I, Burdiles P, Korn O, Csendes P, Henríquez A. Very late results of esophagomyotomy for patients with achalasia: clinical, endoscopic, histologic, manometric and acid reflux studies in 67 patients for a mean follow up of 190 months. Ann Surg 2006; 243: 196-203.

48. Ackroyd R, Watson DI, Devitt PG, Jamieson GG, et al. Laparoscopic cardiomyotomy and partial undoplication for achalasia. Surg Endosc 2001; 15: 983-6.

49. Peracchia A, Bonavina L, Narne S, Segalin A, Antoniazzi 
L, Marotta G. Minimally invasive surgery for Zenker's diverticulum: analysis of results in 95 consecutive patients. Arch Surg 1998; 133: 695-700.

50. Chang CY, Payapilli RJ, Scher RL. Endoscopic staple diverticulostomy for Zenker's diverticulum: review of literature and experience in 159 consecutive cases. Laringoscope 2003; 113: 957-65.

51. Bonavina L, Bettineschi F, Fontebasso V, Segalin A, Peracchia A. Cricopharyngeal myotomy and stapling: treatment of choice for Zenker's diverticulum. In: Nabeya $\mathrm{K}$, Hanaoka T, Nogami H, Eds. Recent advances in diseases of the esophagus. Tokyo, Japan: Springer-Verlag. 1993; 207-11.

52. Aggerholm K, Illum P. Surgical treatment of Zenker's diverticulum. J Laryngol Otol 1990; 104: 312-4.

53. Laing MR, Murthy P, Ah-See KW., Cockburn JS. Surgery for pharyngeal pouch: audit of management with short and long-term follow up. J R Coll Surg Edinb 1995; 40: 315-8.

54. Witterick IJ, Gullane PJ, Yeung E. Outcome analysis of
Zenker's diverticulectomy and cricopharyngeal myotomy. Head Neck 1995; 17: 382-8.

55. Laccourreye O, Menard M, Cauchois R, Huart J, Jouffre V, Brasnu D, Laccourreye H. Esophageal diverticulum: diverticulopexy versus diverticulectomy. Laryngoscope 1994; 104: 889-92.

56. Bonafede JP, Lavertu P, Wood BG, P, Wood BG, Eliachar I. Surgical outcome in 87 patients with Zenker's diverticulum. Laryngoscope 1997; 107: 720-5.

57. Morton RP, Bartley JR. Inversion of Zenker's diverticulum: the preferred option. Head Neck 993; 15: 253-6.

58. Braghetto I, Cardemil G, Schwartz E, Valladares H, Rencoret G, Estay R, et al. Video thoracoscopic management of middle esophageal diverticulum with secondary bronchoesophageal fistula: report a case. Surg today 2008: 38: 1124-8.

59. Braghetto I, Korn O, Valladares H. Divertículos esofágicos: Tratamiento quirúrgico/Epiphrenic diverticula: surgical treatment. Gastroenterol Latinoam 2005; 16: 122-6. 